\title{
Multispectral synthesis of daylight using a commercial digital CCD camera
}

\author{
Juan L. Nieves, Eva M. Valero, Sérgio M. C. Nascimento, Javier Hernández-Andrés, \\ and Javier Romero
}

\begin{abstract}
Performance of multispectral devices in recovering spectral data has been intensively investigated in some applications, as in spectral characterization of art paintings, but has received little attention in the context of spectral characterization of natural illumination. This study investigated the quality of the spectral estimation of daylight-type illuminants using a commercial digital CCD camera and a set of broadband colored filters. Several recovery algorithms that did not need information about spectral sensitivities of the camera sensors nor eigenvectors to describe the spectra were tested. Tests were carried out both with virtual data, using simulated camera responses, and real data obtained from real measurements. It was found that it is possible to recover daylight spectra with high spectral and colorimetric accuracy with a reduced number of three to nine spectral bands. (C) 2005 Optical Society of America

OCIS codes: $\quad 010.1290,150.0150,150.2950$.
\end{abstract}

\section{Introduction}

Multispectral imaging uses a digital charge-coupled device (CCD) camera coupled with colored filters of different spectral bands, ranging from just three components, as in a red-green-blue (RGB) conventional camera, up to hundreds of components, as in a hyperspectral system. ${ }^{1-5}$ The main application is spectral recovery, and therefore these techniques have been applied intensively in areas as diverse as artificial vision, industrial colorimetry, biomedical engineering, and medicine. Of particular interest is their application in atmospheric optics and remote sensing, where it is difficult to obtain high spectral and high spatial resolution at low cost. Traditional telespectroradiometers used in these fields are expensive, only do measurements over limited areas, and may be sequential devices with long scanning times. On the contrary, multispectral image devices, when combined with image-processing techniques, can

J. L. Nieves (jnieves@ugr.es), E. Valero (valerob@ugr.es), J. Hernández-Andrés (javierha@ugr.es), and J. Romero (jromero@ ugr.es) are with Departamento de Óptica, Facultad de Ciencias, Universidad de Granada, 18071 Granada, Spain. S. M. C. Nascimento (smcn@fisica.uminho.pt) is with Departamento de Física, Campus de Gualtar, Universidade do Minho, 4710-057 Braga, Portugal.

Received 3 January 2005; revised manuscript received 3 May 2005; accepted 3 May 2005.

$0003-6935 / 05 / 275696-08 \$ 15.00 / 0$

(C) 2005 Optical Society of America solve portability and cost problems and recover either spectral reflectance or the illumination spectrum at each pixel of a captured image with good spectral resolution.

Although intense research has been focusing in the analysis of multispectral data to recover spectral reflectance functions of objects, e.g., in the area of accurate spectral characterization of artworks, ${ }^{6-8}$ spectral recovery in the context of the spectral power distribution (SPD) of natural illumination has received little attention. Spectral characterization of daylight is important in fundamental research and applications, in particular, for analysis and synthesis of natural scenes. ${ }^{9}$

Linear modeling of SPD of daylight has been applied to allow spectral-daylight recovery by use of a reduced number of parameters and, even for the spiky spectral profiles typical of daylight spectra, the first five eigenvectors suffice to account for more than $99.99 \%$ of the total variance and with only three eigenvectors, it is possible to achieve colorimetrically accurate SPD recoveries. ${ }^{10,11}$ Hernández-Andrés et al. have shown computationally that, with a linear model based on a principal component analysis (PCA) and a selection of ideal Gaussian-type sensors with optimum spectral position and bandwidth, it is possible to recover the spectrum of natural illumination with high spectral and colorimetric accuracy. ${ }^{12} \mathrm{Al}-$ though it is clear from a computational point of view that linear models are adequate for synthesizing daylight, spectral performance in real conditions is un- 


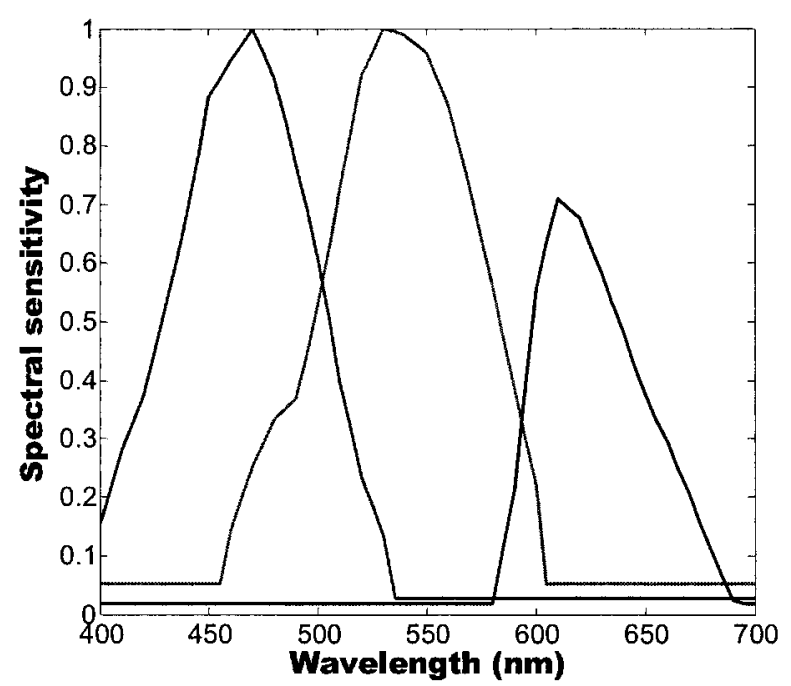

Fig. 1. Spectral sensitivity functions of the Retiga 1300 digital CCD camera from 400 to $700 \mathrm{~nm}$ (sampled at $5 \mathrm{~nm}$ ).

certain because noise can strongly influence the optimization of multispectral devices. ${ }^{13}$

Chiao and coworkers ${ }^{14,15}$ have analyzed a large variety of color signals in natural scenes and characterized the spectra of natural illuminants in forests. They have described a method to recover forest illuminant spectra from the RGB values of a white patch imaged with a digital video camera by using a linear least-squares-fit estimation algorithm. ${ }^{15}$ The performance of the method, however, was limited by noise factors and by the nonideal fitting between the three sensor sensitivities and the eigenvectors derived from PCA of forest illuminant spectra.

The aim in the present work was to investigate the quality of spectral estimation of daylight-type illuminants using a commercial digital CCD camera coupled or not with colored filters. Several recovering techniques based on different algorithms, number, and type of colored filters were tested. Simulated camera responses and real measurements were used. It was found that it is possible to recover daylight spectra with high spectral and colorimetric accuracy with a reduced number of spectral bands.

\section{Spectral Daylight Estimation Using Simulated Camera Responses}

The digital camera used was a Retiga 1300 , with a spatial resolution of $1280 \times 1024$ pixels and a digital resolution of 12 bits per channel $(6.7 \mu \mathrm{m} \times 6.7 \mu \mathrm{m}$ pixel size, with $2 / 3$ in. optical format, and sensor readout noise of 8 electrons $\mathrm{rms}$ ). The sensitivities of the camera were obtained from Quantitative Imaging Corporation and are represented in Fig. 1.

In the simulations of the camera's response, we assumed that it was imaging a uniform white reference surface with spectral reflectance function $r_{w}(\lambda)$. Camera responses were estimated as follows. Let $k$ be the number of sensors that made the array of camera sensors, $Q_{k}(\lambda)$ be the spectral sensitivity of the $k$ th sensor (assumed to include the camera lens' spectral

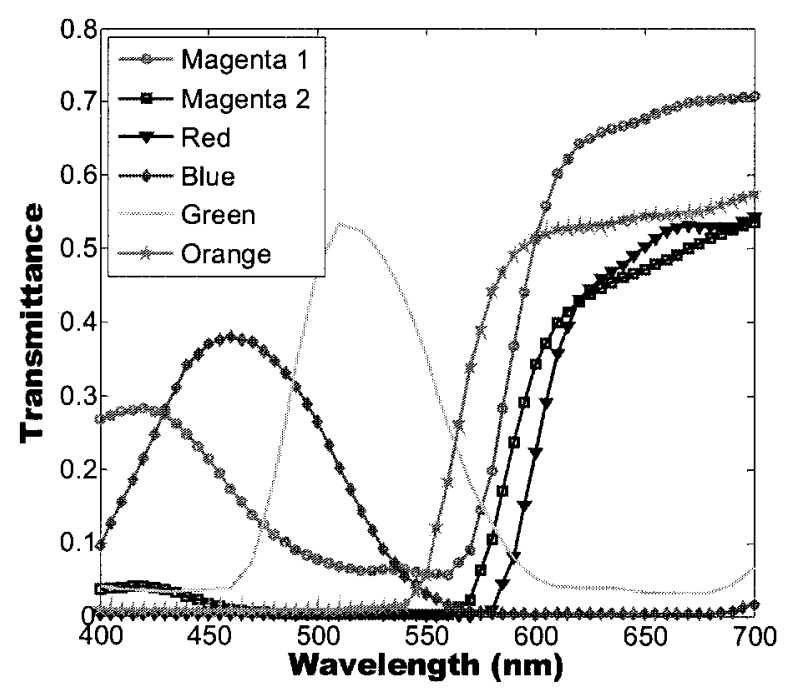

Fig. 2. Spectral transmittance curves of the six color filters used for the simulated and for the experimental wide-band imaging SPD synthesis.

transmittance effects), and $E(\lambda)$ be the SPD of the illuminant impinging on the white reference surface. The response of the $k$ th sensor $\rho_{k}$, which will be assumed linear, is

$$
\rho_{k}=\sum_{\lambda=400}^{700} E(\lambda) r_{W}(\lambda) Q_{k}(\lambda)
$$

The achromatic patch number 19 from the GretagMacBeth ColorChecker ${ }^{16}$ was selected as the white reference surface. The camera responses were computed for a set of 433 SPDs of daylight-type illuminants that were previously measured in our laboratory. ${ }^{11}$ These SPD measurements were made for clear-sky conditions from noon to sunset, including civil twilight, and covered a range of correlated color temperatures from $5000 \mathrm{~K}$ to $11000 \mathrm{~K}$.

Camera responses were computed assuming the CCD camera coupled with different sets of broadband color filters. Thus, data were obtained with a single a set of $k=3$ sensors, the three native RGB channels of the camera, a set of $k=6$ sensors with three filtered RGB channels, and a set of $k=9$ sensors with six filtered RGB channels. The spectral transmittance curves of the set of six broadband gelatin color filters used are shown in Figure 1.

\section{A. Eigenvector Analysis with Pseudoinverse Transformation}

It is possible to find square-integrable functions $V_{i}(\lambda)(i=1,2, \ldots m)$ such that for any SPD of the illuminant there is a single set of real numbers, $\varepsilon_{i}$, such that ${ }^{17}$

$$
E(\lambda)=\sum_{i=1}^{m} \varepsilon_{i} V_{i}(\lambda)
$$

where we have assumed that the illumination is spatially uniform over the white reference surface. Most 
of the SPD of natural illuminants can be described by small-dimensional linear models, and earlier studies have shown that three to five eigenvectors, which can be obtained by PCA, suffice for adequate reconstructions of illuminants. ${ }^{10,11}$ By incorporating Eq. (2) into Eq. (1), the response of the $k$ sensors can be expressed in matrix notation as

$$
\boldsymbol{\rho}=\boldsymbol{\Lambda} \cdot \boldsymbol{\varepsilon},
$$

where

$$
\Lambda_{k i}=\sum_{\lambda} V_{i}(\lambda) r_{W}(\lambda) Q_{k}(\lambda),
$$

and the coefficients $\varepsilon_{i}$ are calculated by the orthogonal projection,

$$
\varepsilon_{i}=\left\langle E(\lambda) \mid V_{i}(\lambda)\right\rangle .
$$

If the number of sensors equals the number of eigenvectors that are used to linearly represent each of the SPDs in a suitable base, e.g., $k=m$, Eq. (3) can be solved for $\varepsilon$ by inverting the matrix $\Lambda$. But recovery is also possible when the number of sensors does not equal the number of eigenvectors. ${ }^{17}$ In this case the simulated camera responses $\rho_{k}$ can be used to create a pseudoinverse transform of $\boldsymbol{\Lambda}$ that converts camera responses to eigenvector coefficients of illuminants. ${ }^{1}$ A two-step $m \times k$ transformation is derived by fitting the $k$ simulated digital signals and the $m$ eigenvector coefficients, $\varepsilon_{i}$, obtained from Eq. (5). Analysis of the influence of the choice of eigenvectors $V_{i}(\lambda)$ both on the reconstruction errors and on the ill-conditioness of the matrix $\Lambda$ has shown that eigenvectors obtained from a set of 2600 daylight spectra ${ }^{11}$ produced a high degree of spectral and colorimetric accuracy. ${ }^{12}$ Thus, in the following calculations we have used the eigenvectors $V_{i}(\lambda)$ obtained by PCA from that set. In order to obtain the estimated spectrum $\mathbf{E}_{e}$ from $\boldsymbol{\rho}$, let $\mathbf{G}$ be the $m \times k$ matrix operating from the right-hand side such that

$$
\boldsymbol{\varepsilon}_{e}=\mathbf{G} \cdot \boldsymbol{\rho},
$$

where $\boldsymbol{\varepsilon}_{e}$ are the estimated $m$ eigenvector coefficients. A relationship between $\boldsymbol{\varepsilon}_{e}$ and digital counts $\boldsymbol{\rho}$ can be established by the usual pseudoinverse calculation, and the matrix $\mathbf{G}$ is therefore expressed as

$$
\mathbf{G}=\boldsymbol{\varepsilon} \boldsymbol{\rho}^{T}\left[\boldsymbol{\rho} \cdot \boldsymbol{\rho}^{T}\right]^{-1} .
$$

Combining Eqs. (6) and (7), the estimated SPD of illuminants can finally be obtained from the matrix product,

$$
\mathbf{E}_{e}=\mathbf{V}(\mathbf{G} \cdot \boldsymbol{\rho}) .
$$

The method requires an adequate choice of the $k$ sensors. Although the types of sensors are determined by the digital CCD camera selected, the use of different colored filters placed in front of the camera lens will determine their number. The number of sensors determines the dimension of the base derived from PCA and influences the spectral and colorimetric quality of the SPD estimation.

\section{B. Direct Pseudoinverse Transformation}

This method consists on establishing a direct relationship between the camera responses and the SPD of the illuminants. It is based on a one-step $61 \times k$ transformation that is derived by minimizing the least-square error between the original $E(\lambda)$ and the estimated SPD of the illuminants $E_{e}(\lambda)$ from the $k$ digital signals. This means that a transformation matrix is directly obtained from the spectra of the 433 SPDs of daylight-type illuminants and the $k$ digital signals.

Based on a least-squared estimation, the error defined by

$$
\text { error }=\|\mathbf{E}-\mathbf{F} \cdot \boldsymbol{\rho}\|^{2},
$$

where $\mathbf{F} \cdot \boldsymbol{\rho}$ represents the estimated $\mathrm{SPD}$, is minimized with respect to $\mathbf{F}$, and we have

$$
\mathbf{F}=\mathbf{E} \cdot \boldsymbol{\rho}^{T}\left[\boldsymbol{\rho} \cdot \boldsymbol{\rho}^{T}\right]^{-1} .
$$

The spectrum of the estimated illuminant is directly obtained as

$$
\mathbf{E}_{e}=\mathbf{E} \cdot \boldsymbol{\rho}^{T}\left[\boldsymbol{\rho} \cdot \boldsymbol{\rho}^{T}\right]^{-1} \boldsymbol{\rho} .
$$

Thus, using this method, the SPD of illuminants does not need to be described in any mathematical base.

\section{Metrics for Spectral and Colorimetric Quality Evaluation}

Spectral estimation is a multidimensional problem, and a combination of different metrics should be used to avoid mononumerosis, as a single number may not capture variations that may be significant. In this work three metrics were used. The spectral curves were compared using the root-mean-square error (RMSE) and the goodness-of-fit coefficient ${ }^{11,18}$ (GFC). The GFC is based on the Schwartz's inequality, and its value is in the range $0-1$. Colorimetrically accurate daylight estimations require GFC $>0.995$, GFC $>0.999$ indicates a quite good spectral fit, and values GFC > 0.9999 signifies an almost-exact fit. The CIELab color difference formula was used to evaluate the colorimetric quality, and differences of less than 3 CIELab units between the original and the estimated spectra were considered acceptable. ${ }^{19-21}$

\section{Results}

The original spectra and chromaticities were compared with those recovered from simulated camera responses. Figure 3 shows examples of the 99th percentile for GFC and for $\Delta E_{a b} *$ for the estimation algorithms tested for the camera without filter, $k=3$, 

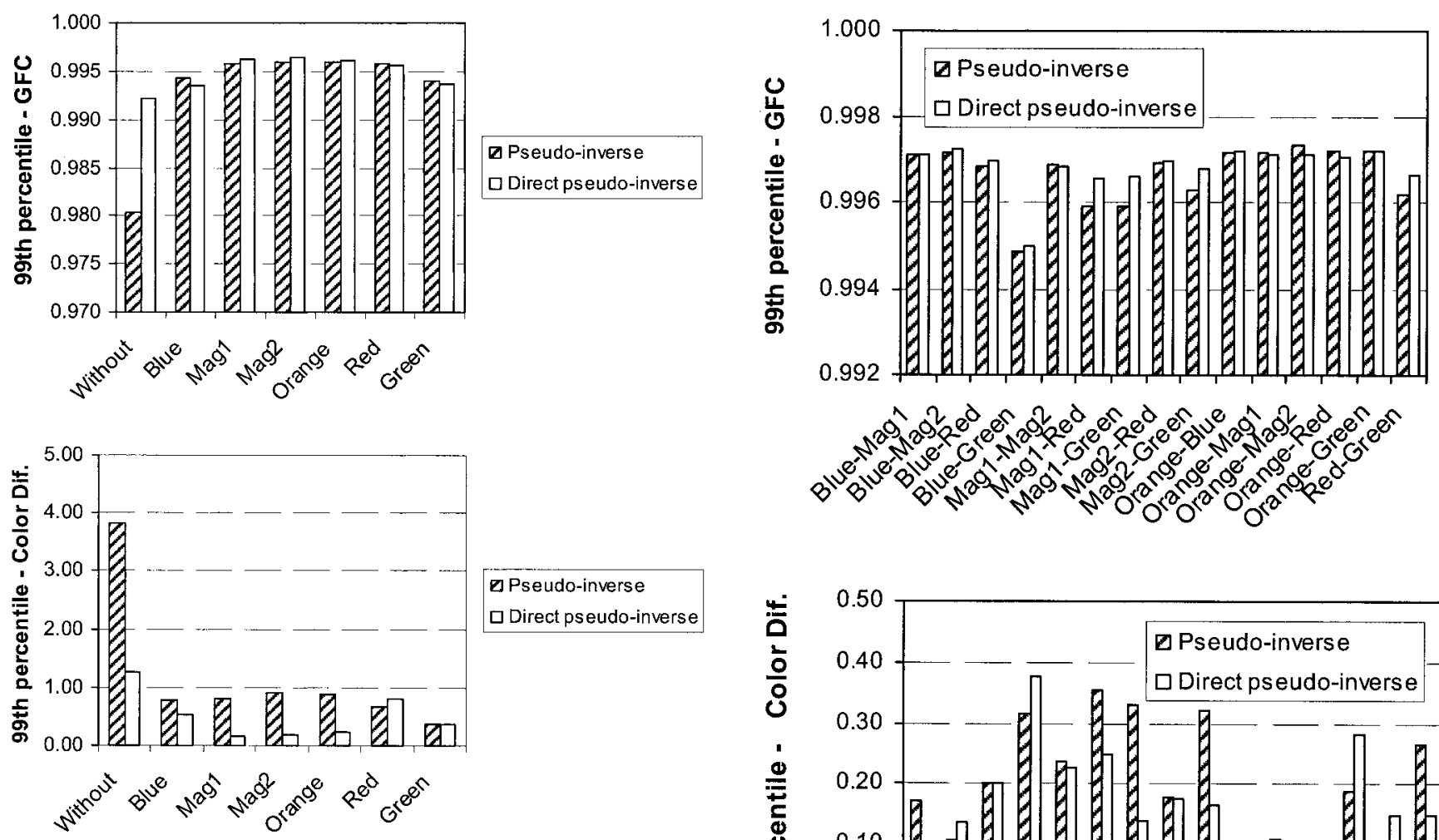

Fig. 3. 99th percentile for GFC (upper figure) and for CIELab units (lower figure) for the estimation algorithms tested for the camera without filter, $k=3$, and for each of single colored filters, $k=6$. Data obtained from the synthesis methods with the same number of sensors and eigenvector coefficients.

and for each of single colored filters, $k=6$. Estimation of SPD is accurate for all cases, with 99th percentile for GFC around 0.995, except for the pseudoinverse with 3 bands, without color filter, with a GFC value of 0.9974 on average and color difference clearly over 1 CIELab unit. The direct pseudoinverse method produced, in general, the most accurate results with the filter magenta2.

Figure 4 shows examples of the 99 th percentile for GFC and for CIELab and the different sets of double colored filters used, $k=9$; the results shown for the
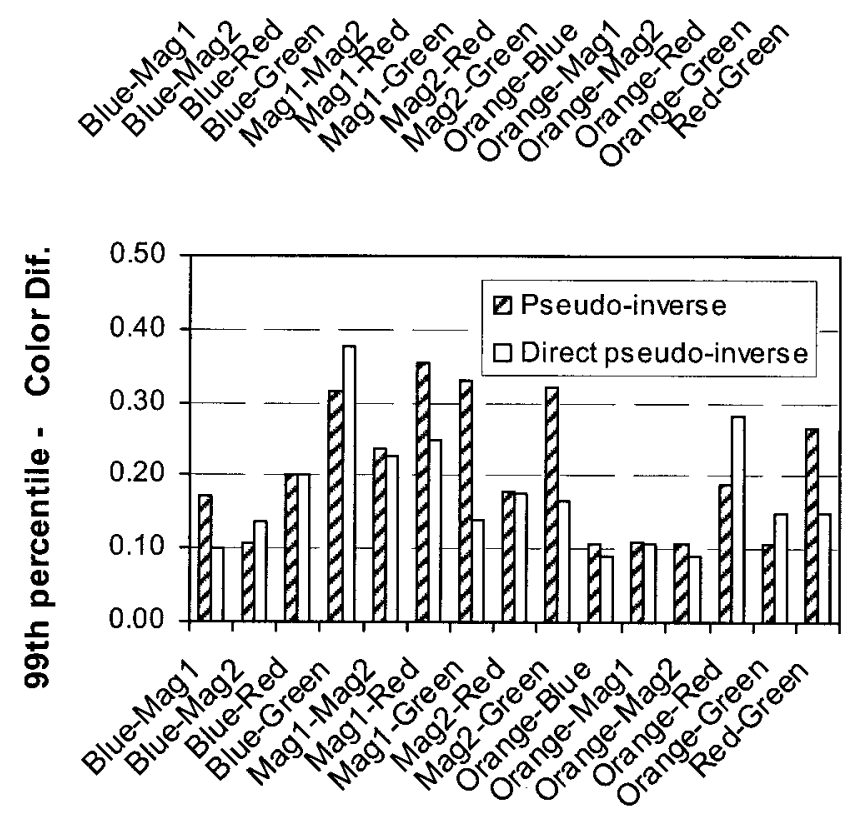

Fig. 4. 99th percentile for GFC (upper figure) and for CIELab units (lower figure) for the estimation algorithms and for the different sets of double colored filters used, $k=9$. Data obtained from the synthesis methods with the same number of sensors and eigenvector coefficients.

pseudoinverse transformation are for an equal number of sensors and eigenvectors. As expected, accuracy is better than that obtained for the camera without filter or with one single colored filter. Spec-

Table 1. Mean, Standard Deviation, and 99th Percentile Results Obtained for Simulated Digital Counts in Recovering 433 SPD Daylight Spectra

\begin{tabular}{|c|c|c|c|c|c|c|c|c|c|c|c|}
\hline \multirow[b]{2}{*}{ Algorithm } & \multirow[b]{2}{*}{$\begin{array}{c}\text { Filter } \\
\text { Combination }\end{array}$} & \multirow[b]{2}{*}{$\begin{array}{l}\text { Number of } \\
\text { Eigenvectors }\end{array}$} & \multicolumn{3}{|c|}{ GFC } & \multicolumn{3}{|c|}{ RMSE } & \multicolumn{3}{|c|}{$\Delta E_{a b}{ }^{*}$} \\
\hline & & & Mean & Std & $\begin{array}{l}\text { 99th } \\
\text { Perc. }\end{array}$ & Mean & Std & $\begin{array}{l}\text { 99th } \\
\text { Perc. }\end{array}$ & Mean & Std & $\begin{array}{l}\text { 99th } \\
\text { Perc. }\end{array}$ \\
\hline \multirow[t]{6}{*}{ Pseudoinverse } & Without & 3 & 0.9974 & 0.0049 & 0.9804 & 0.0061 & 0.0068 & 0.0253 & 0.9376 & 1.0325 & 3.8267 \\
\hline & & 6 & 0.9975 & 0.0048 & 0.9804 & 0.0060 & 0.0067 & 0.0253 & 0.9375 & 1.0325 & 3.8266 \\
\hline & & 9 & 0.9996 & 0.0012 & 0.9943 & 0.0020 & 0.0028 & 0.0136 & 0.1475 & 0.2278 & 1.1519 \\
\hline & Mag2 & 6 & 0.9998 & 0.0008 & 0.9960 & 0.0015 & 0.0021 & 0.0115 & 0.1339 & 0.1895 & 0.9142 \\
\hline & & 9 & 0.9998 & 0.0008 & 0.9961 & 0.0012 & 0.0021 & 0.0112 & 0.0339 & 0.0678 & 0.3036 \\
\hline & Orange-Mag2 & 9 & 0.9999 & 0.0007 & 0.9973 & 0.0009 & 0.0017 & 0.0094 & 0.0146 & 0.0176 & 0.1058 \\
\hline \multirow{3}{*}{$\begin{array}{l}\text { Direct } \\
\quad \text { Pseudoinverse }\end{array}$} & Without & & 0.9995 & 0.0016 & 0.9922 & 0.0017 & 0.0035 & 0.0160 & 0.1064 & 0.2382 & 1.2568 \\
\hline & Mag2 & & 0.9998 & 0.0008 & 0.9965 & 0.0009 & 0.0021 & 0.0107 & 0.0171 & 0.0379 & 0.1753 \\
\hline & Orange-Mag2 & & 0.9999 & 0.0006 & 0.9971 & 0.0007 & 0.0018 & 0.0097 & 0.0081 & 0.0204 & 0.0919 \\
\hline
\end{tabular}


tral accuracy for both eigenvector analysis with pseudoinversion and direct pseudoinversion methods are similar with average GFC $( \pm \mathrm{SD})$ of 0.99985 \pm 0.00005 and $0.99987 \pm 0.00004$, respectively, indicating very good spectral fits. Colorimetric accuracy, however, is a bit better for the direct pseudoinverse method with average CIELab units of $\Delta E_{a b} *=0.0148 \pm 0.0073$ and $0.0246 \pm 0.0086$, respectively. Note that these color differences are clearly below 1 CIELab unit, which means that original and estimated SPD are visually indistinguishable.

An increase in the number of sensors enhances both the spectral and the colorimetric performance of the SPD recovery, independently of the estimation method used. This has been established in previous works for spectral recovery of reflectances and daylights using simulated sensors when noise effects are not included. ${ }^{3,12}$ Nevertheless, a recent study $^{22}$ shows that this could not be the case when different kinds of noise are considered; in this case, increasing the number of sensors and/or the number of eigenvector does not necessarily improve the accuracy of estimated spectra in the multispectral systems.

When the direct pseudoinverse transformation is used, the average GFC for all filter combinations was 0.9997 and 0.9999 when six and nine sensors were used, respectively, and the mean GFC for the unfiltered RGB signals was 0.9995 ; in any case, the results indicate a very good spectral recovery and an almost perfect colorimetric one. This also agrees with the Imai and co-workers findings when surface reflectances are imaged using trichromatic digital cameras. ${ }^{1}$ The pseudoinverse transformation for six sensors is less sensitive to the number of eigenvectors, with the colorimetric accuracy being very similar in this case (the 99th percentile for CIELab units is $\Delta E_{a b} *=0.9142$ for six versus 0.3036 for nine eigenvectors).

According to these results, the filter pair magenta2-orange was selected as the most appropriate filter combination for the nine-band SPD estimation. Table 1 summarizes the mean, standard deviation, and 99th percentile results of the SPD's recovery for the filter and filter combination selected, which will be used in the next section; the table also shows the performance of the eigenvector analysis with different number of eigenvectors. The results of the eigenvector analysis with pseudoinverse reinforces the idea of avoiding the mononumerosis to evaluate the quality of the SPD recoveries because the spectral accuracy does not change appreciably when the number of eigenvectors increases; however, the colorimetric accuracy improves when nine eigenvectors is used. The direct pseudoinverse transformation is more accurate than the eigenvector analysis with the pseudoinverse method. Figure 5 shows examples of SPD recoveries with 99th percentile GFC values obtained with the direct pseudoinverse algorithm for three-, six-, and nine-band SPD estimation. Results are for daylights measured previously at sun-
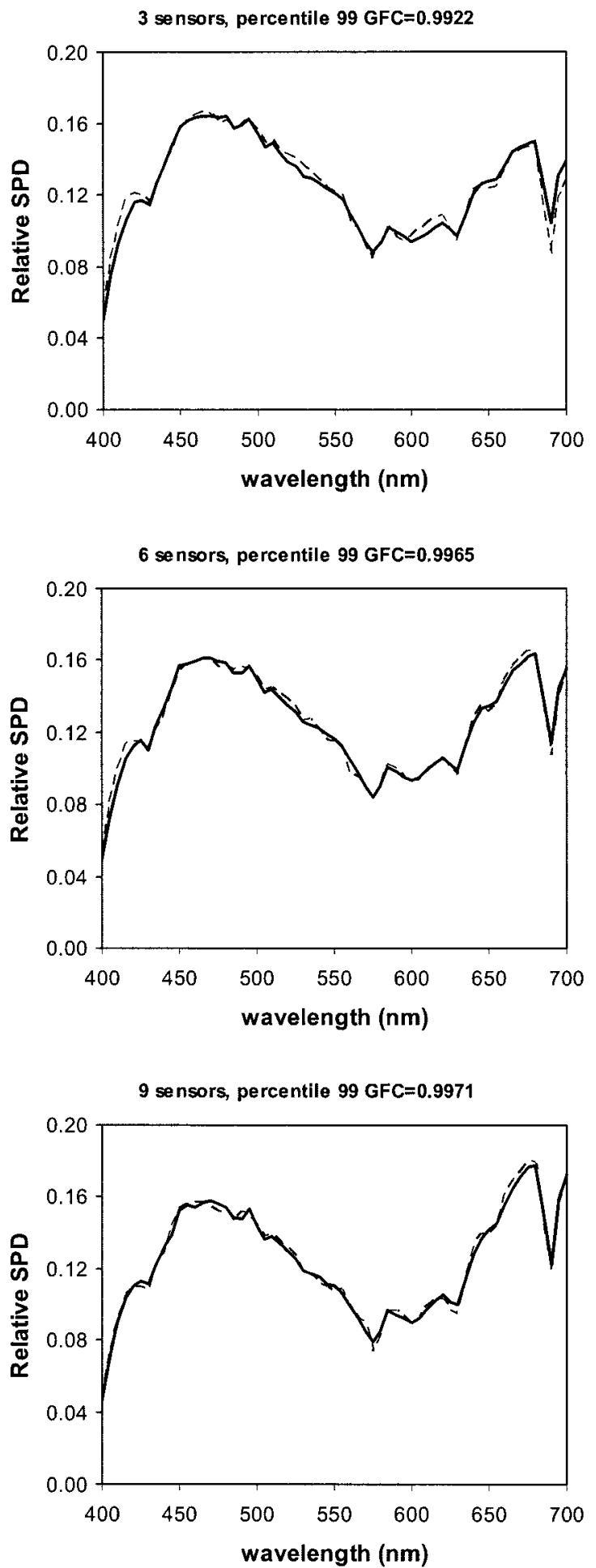

Fig. 5. Original daylight spectrum (solid curve) and recovered spectrum (dashed curve) using the direct pseudoinverse method with simulated digital counts and three, six, and nine sensors. Results are for the 99th percentile of the GFC, and the original SPDs correspond to sunset hours of a day.

set in our laboratory; the spectral profile of these SPDs shows the typical absorption bands and relative high spectral content at large wavelengths. But even with only three sensors, this kind of daylight 
Table 2. Mean, Standard Deviation, and 99th Percentile Results Obtained for Measured Digital Counts in Recovering 30 SPD Daylight Spectra

\begin{tabular}{|c|c|c|c|c|c|c|c|c|c|c|c|}
\hline \multirow[b]{2}{*}{ Algorithm } & \multirow[b]{2}{*}{$\begin{array}{c}\text { Filter } \\
\text { Combination }\end{array}$} & \multirow[b]{2}{*}{$\begin{array}{l}\text { Number of } \\
\text { Eigenvectors }\end{array}$} & \multicolumn{3}{|c|}{ GFC } & \multicolumn{3}{|c|}{ RMSE } & \multicolumn{3}{|c|}{$\Delta E_{a b} *$} \\
\hline & & & Mean & Std & $\begin{array}{l}\text { 99th } \\
\text { Perc. }\end{array}$ & Mean & Std & $\begin{array}{l}\text { 99th } \\
\text { Perc. }\end{array}$ & Mean & Std & $\begin{array}{l}\text { 99th } \\
\text { Perc. }\end{array}$ \\
\hline \multirow[t]{5}{*}{ Pseudoinverse } & Without & 3 & 0.9961 & 0.0040 & 0.9892 & 0.0099 & 0.0055 & 0.0187 & 1.0820 & 0.3350 & 1.7569 \\
\hline & & 6 & 0.9975 & 0.0032 & 0.9921 & 0.0073 & 0.0056 & 0.0161 & 0.5770 & 0.5972 & 1.6360 \\
\hline & & 9 & 0.9974 & 0.0031 & 0.9922 & 0.0074 & 0.0055 & 0.0160 & 0.7279 & 0.6526 & 1.8651 \\
\hline & Mag2 & 6 & 0.9976 & 0.0029 & 0.9929 & 0.0071 & 0.0053 & 0.0153 & 0.5590 & 0.5780 & 1.5260 \\
\hline & Orange-Mag2 & 9 & 0.9974 & 0.0033 & 0.9916 & 0.0073 & 0.0058 & 0.0166 & 0.5580 & 0.5960 & 1.6450 \\
\hline \multirow{3}{*}{$\begin{array}{l}\text { Direct } \\
\quad \text { Pseudoinverse }\end{array}$} & Without & & 1.0000 & 0.0000 & 0.9998 & 0.0011 & 0.0006 & 0.0024 & 0.1743 & 0.0855 & 0.3985 \\
\hline & Mag2 & & 1.0000 & 0.0000 & 0.9998 & 0.0010 & 0.0007 & 0.0023 & 0.1737 & 0.1134 & 0.4986 \\
\hline & Orange-Mag2 & & 0.9999 & 0.0001 & 0.9997 & 0.0011 & 0.0009 & 0.0031 & 0.1902 & 0.1491 & 0.5086 \\
\hline
\end{tabular}

spectra is well recovered with a colorimetric accuracy of only $\Delta E_{a b} *=1.2568$.

\section{Spectral Daylight Estimation Using Measured Camera Response}

\section{A. Methods}

To estimate the quality of the spectral recovery in real conditions, the achromatic patch number 19 of the GretagMacBeth ColorChecker was imaged outdoors under different weather conditions and hours of the day using the same digital camera as for the simulated digital counts. Simultaneously, the color signal from this patch was measured with a telespectroradiometer PR-650. All measurements were made between noon and sunset from 12:00 to 19:00 hours local time (UTC +1 hour). The spectral reconstruction of the SPD from the achromatic patch was computed using three, six, and nine bands. Given the results described above, the magenta 2 and the pair magenta2-orange were the filter and filter combinations selected.

In each measurement the achromatic patch was recorded with three, six, and nine digital signals, corresponding to the filter configurations described. The ColorChecker was held at a $45^{\circ}$ angle and $50 \mathrm{~cm}$ above the floor. The camera and the telespectroradiometer were placed in locations where the illuminant was uniformly distributed over the color chart. The specular component of direct sunlight was always avoided for all measurements. Dark images were obtained with the camera lens caps on to compensate for pattern noise from the camera, the camera was linear for the conditions of the measurements, and the exposure times were adjusted for each image and filter combination. It should be noted that the illuminant spectra outdoors vary mainly in luminance, and the only marked spectral changes are appreciated at sunset. Transformation matrix $\mathbf{G}$ was derived from a subset of 20 SPDs, and an additional set of 10 SPDs was used to test the multispectral synthesis algorithms.

\section{B. Results}

The original spectra and chromaticities were compared with those recovered from measured camera responses. Table 2 shows the mean, standard deviation, and 99th percentile results for the SPD recoveries using three, six, and nine bands for the two synthesis methods; the table also shows the performance of the eigenvector analysis with different numbers of eigenvectors. As in previous computational results, increasing the number of eigenvectors improves the colorimetric accuracy, but a small improvement is obtained in the spectral accuracy when the pseudoinverse method is used. This is more evident for three and nine eigenvectors with $\Delta E_{a b}$ * $=1.0820$ and 0.7279 , respectively.

Direct pseudoinverse gives better spectral and colorimetric accuracy than the pseudoinverse SPD synthesis method (average GFC value of 0.9999 versus 0.9970 ). The colorimetric accuracy increases on average from $\Delta E_{a b}{ }^{*}=0.7330$ for pseudoinverse to 0.1794 for the direct-pseudoinverse method. Also increasing the number of sensors reduces the errors in the estimated SPDs, although the colorimetric accuracy is slightly better for six than for nine bands. All the estimation errors are very similar when the pseudoinverse method is used; these differences correspond to very small spectral differences as can be seen in Fig. 6, where the original and the estimated SPD are plotted for different numbers of bands and the direct pseudoinverse method. The differences are of little importance and suggest that the number of bands could be reduced depending on the technical application. It is a matter of further studies to analyze the appropriateness of transformation matrix $\mathbf{G}$ for different spectral and spatial resolution when the number of bands changes.

\section{Discussion and Conclusions}

Algorithms to estimate SPDs of either natural daylight-type or artificial illuminants generally assume linear modeling of spectra and use a reduced number of parameters (three to five) to recover the 

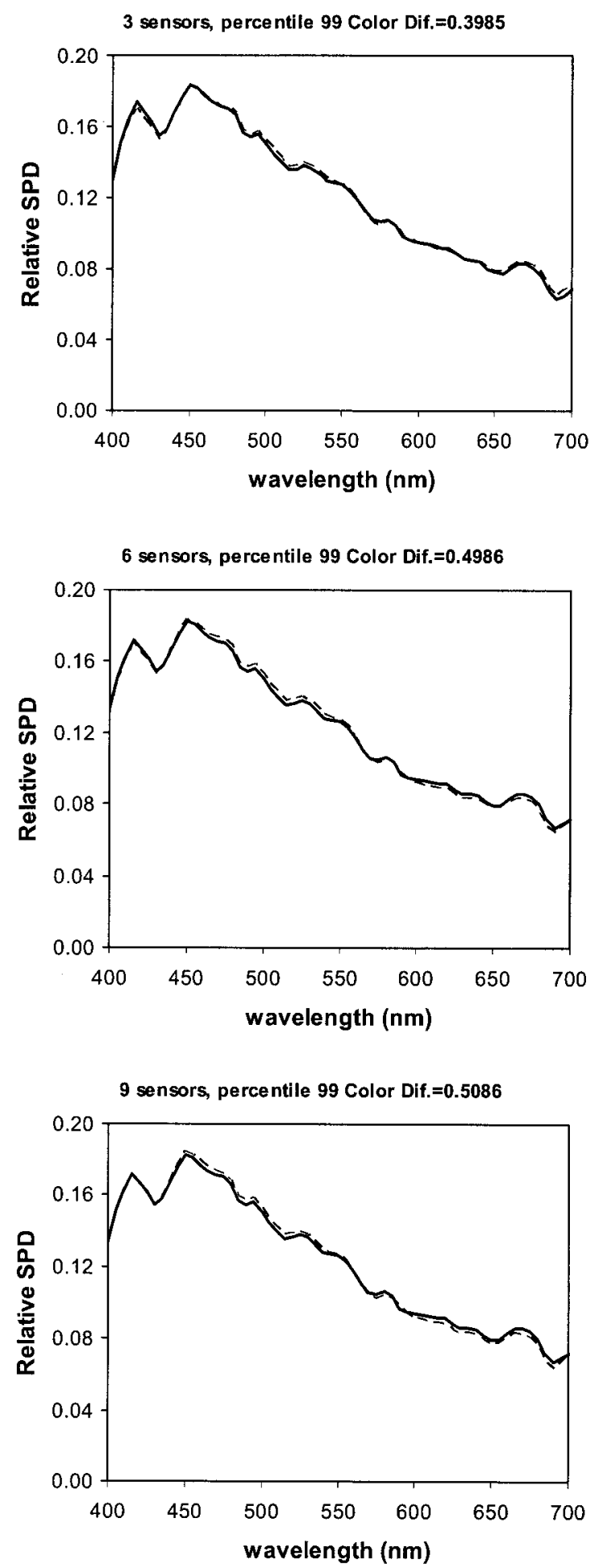

Fig. 6. Original daylight spectrum measured in Granada at noon (solid curve) and recovered spectrum (dashed curve) using the direct pseudoinverse method with three, six, and nine sensors and real measurements; results are for the 99th percentile of the CIELab units.

spectral profile of illuminants with spectral and colorimetric accuracy. ${ }^{10,11}$ Also, the computational design of a multispectral device to estimate the SPD of daylights with a reduced number of both simulated
Gaussian and commercial sensors has been proposed. ${ }^{12,22}$

In this paper a multispectral device to synthesize SPDs of daylight-type illuminants with high spectral and colorimetric accuracy is proposed. Based on similar techniques developed for spectral image capture of art paintings, ${ }^{1,6}$ the algorithm is based on a priori analysis of an RGB set of signals from a white surface captured by a digital CCD camera, and it increases the number of sensors by using colored filters in front of the camera lens. The spectral synthesis improves as the number of RGB signals increases for the filter combinations used. When comparing these spectral recoveries with either an earlier computational approach or experimental ones, ${ }^{12,15}$ the present synthesis algorithm confirms the CCD's potential as a daylight-estimation device even with the presence of noise. When a commercial CCD camera was simulated, a 99th percentile of 0.9921 was obtained for the GFC; in the present study we have obtained a value of 0.9916 with the direct pseudoinverse, which is very close to the one obtained computationally.

Results suggest that direct pseudoinverse surpasses eigenvector analysis with simple inversion and pseudoinverse transformation for both virtual data using simulated digital counts and real measurements. The differences obtained for spectral and colorimetric accuracy between pseudoinverse and direct pseudoinverse methods decrease particularly for six and nine sensors and measured digital counts. Even with six sensors the colorimetric accuracy is very good and slightly better than for nine sensors. Thus, the spectral estimation of daylight-type illuminants using a trichromatic digital camera and $a$ priori analysis of spectral properties is plausible not only for reflectances but for SPD of illuminants also.

The great advantage of this synthesis algorithm by comparison with other algorithms using CCD camer$\operatorname{as}^{15}$ is that it does not need information about spectral sensitivities of the camera sensors. Thus, the performance of the direct pseudoinverse method does not depend either on possible ill-conditioness of the transformation matrices derived from eigenvector analysis with simple inversion methods [see Eq. (3)] or on the selected illuminant spectral used in the eigenvector analysis. The method proposed here is optimized with appropriate selection of the training set SPDs; the larger the set of different SPDs of illuminants, including different atmospheric conditions, hours of the day and seasons, the better spectral and colorimetric accuracy will be.

We thank Francisco H. Imai for comments and suggestions. This work was supported by the Comisión Interministerial de Ciencia y Tecnología (CICYT), Spain, under research grant DPI 2004-03734.

\section{References}

1. F. H. Imai and R. Berns, "Spectral estimation using trichromatic digital cameras," in International Symposium on Multispectral Imaging and Color Reproduction for Digital Archives (Society of Multispectral Imaging of Japan, 1999), pp. 42-49. 2. F. H. Imai, R. Berns, and D-Y. Tzeng, "A comparative analysis 
of spectral reflectance estimated in various spaces using a trichromatic camera system," J. Imaging Sci. Technol. 44, 280-371 (2000).

3. D. Connah, S. Westland, and M. G. A. Thompson, "Recovering spectral information using digital camera systems," Color Technol. 117, 309-312 (2001).

4. J. Hardeberg, F. Schmitt, and H. Brettel, "Multispectral color image capture using a liquid crystal tunable filter," Opt. Eng. 41, 2532-2548 (2002).

5. B. Hill, "(R)Evolution of color imaging systems," in Proceedings of the Second European Conference on Colour in Graphics, Imaging and Vision (Society for Imaging Science and Technology, Springfield, Va., 2004), pp. 473-479.

6. F. H. Imai and R. S. Berns, "Spectral estimation of oil paints using multi-filter trichromatic imaging," in Proceedings of the 9th Congress of the International Colour Association (Proc. SPIE 4421, 504-507 (2002).

7. Y. Murakami, T. Obi, M. Yamaguchi, N. Ohyama and Y. Komiya, "Spectral reflectance estimation from multi-band image using color chart," Opt. Commun. 188, 47-54 (2001).

8. R. S. Berns, L. A. Taplin, F. H. Imai, E. A. Day, and D. C. Day, "Spectral imaging of Matisse's Pot of Geraniums: a case study," in Proceedings of Eleventh Color Imaging Conference: Color Science and Engineering, Systems, Technologies and Applications (IS\&T, Springfield, 2003) pp. 149-153.

9. S. M. C. Nascimento, F. P. Ferreira, and D. H. Foster, "Statistics of spatial cone-excitation ratios in natural scenes," J. Opt. Soc. Am. A 19, 1484-1490 (2002).

10. J. Romero, A. García-Beltrán, and J. Hernández-Andrés, "Linear bases for representation of natural and artificial illuminants," J. Opt. Soc. Am. A 14, 1007-1014 (1997).

11. J. Hernández-Andrés, J. Romero, J. L. Nieves, and R. L. Lee, Jr., "Color and spectral analysis of daylight in southern Europe," J. Opt. Soc. Am. A 18, 1325-1335 (2001).

12. J. Hernández-Andrés, J. L. Nieves, E. Valero, and J. Romero, "Spectral-daylight recovery by use of only a few sensors," J. Opt. Soc. Am. A 21, 13-23 (2004).
13. D. Conahh, S. Westland, and M. G. A. Thompson, "Optimization of a multispectral imaging system," in Proceedings of the First European Conference on Colour in Graphics, Imaging and Vision (Society for Imaging Science and Technology, Springfield, Va., 2002), pp. 619-622.

14. C.-C. Chiao, T. W. Cronin, and D. Osorio, "Color signals in natural scenes: characteristics of reflectance spectra and effects of natural illuminants," J. Opt. Soc. Am. A 17, 218-224 (2000).

15. C.-C. Chiao, D. Osorio, M. Vorobyev, and T. W. Cronin, "Characterization of natural illuminants in forests and the use of digital video data to reconstruct illuminant spectra," J. Opt. Soc. Am. A 17, 1713-1721 (2000).

16. ColorChecker Chart from GretagMacbeth Ltd. ("GMB") (2004).

17. L. T. Maloney and B. Wandell, "Color constancy: a method for recovering surface spectral reflectance," J. Opt. Soc. Am. A 3, 23-33 (1986).

18. F. H. Imai, M. R. Rosen, and R. S. Berns, "Comparative study of metrics for spectral match quality," in Proceedings of the First European Conference on Colour in Graphics, Imaging and Vision (Society for Imaging Science and Technology, Springfield, Va., 2002), pp. 492-496.

19. M. J. Vrhel, R. Gershon, and L. S. Iwan, "Measurement and analysis of object reflectance spectra," Color Res. Appl. 19, 4-9 (1994).

20. G. Finlayson, "Spectral sharpening: what is it and why is it important," in Proceedings of the First European Conference on Colour in Graphics, Imaging and Vision (Society for Imaging Science and Technology, Springfield, Va., 2002), pp. 230-235.

21. National Institute of Standards and Technology (NIST). http: //physics.nist.gov/Divisions/Div844/Newrad/abstracts/Nadal Poster.htm.

22. M. A. López-Álvarez, J. Hernández-Andrés, J. Romero and R. L. Lee, Jr., "Designing a practical system for spectral imaging of skylight," Appl. Opt. 44, 5688-5695 (2005). 